\title{
Computational cost for detecting inspiraling binaries using a network of laser interferometric detectors
}

\author{
Archana Pai $\dagger$, Sukanta Bose $\$ \S$, and Sanjeev Dhurandhar $\dagger$ \\ $\dagger$ Inter-University Centre for Astronomy and Astrophysics, Pune, India \\ $\ddagger$ Department of Physics and Program in Astronomy, Washington State University, \\ Pullman, WA 99164-2814, USA \\ $\S$ Max Planck Institut für Gravitationphysik, Albert Einstein Institut, Am \\ Mühlenberg 1, Golm, D-14476, Germany \\ E-mail: apai@iucaa.ernet.in, sukanta@wsu.edu, sanjeev@iucaa.ernet.in
}

\begin{abstract}
We extend a coherent network data-analysis strategy developed earlier for detecting Newtonian waveforms to the case of post-Newtonian (PN) waveforms. Since the PN waveform depends on the individual masses of the inspiraling binary, the parameter-space dimension increases by 1 from that of the Newtonian case. We obtain the number of templates and estimate the computational costs for PN waveforms: For a lower mass limit of $1 M_{\odot}$, for LIGO-I noise, and with $3 \%$ maximum mismatch, the online computational speed requirement for single detector is a few Gflops; for a two-detector network it is hundreds of Gflops and for a three-detector network it is tens of Tflops. Apart from idealistic networks, we obtain results for realistic networks comprising of LIGO and VIRGO. Finally, we compare costs incurred in a coincidence detection strategy with those incurred in the coherent strategy detailed above.
\end{abstract}

PACS numbers: 04.80 Nn, 07.05 Kf, 97.80 -d 


\section{Introduction}

Close compact binaries are among the prime sources of gravitational waves that hold promise for detection with upcoming laser interferometric detectors such as LIGO, VIRGO, GEO-600, TAMA, and AIGO. The back-reaction of radiated gravitational waves results in an inspiral with an eventual merger of the two companions of the binary system. This adiabatic inspiral waveform has been accurately modeled upto 2.5 postNewtonian order (PN) [1]. In an earlier work [2], we developed a formalism for detecting inspiral waveform with a network of detectors. The proposed analysis is of coherent nature where the network is treated as a single detector and the data is combined using the phase information optimally. In [2], we used the maximum likelihood detection (MLD) technique, which involves correlating the output of a network of detectors with the family of expected waveforms (or templates) and selecting the maximum of the network likelihood ratio for decision making [3]. To reduce computational costs involved in searching over the space of source parameters, we succeeded in analytically maximizing over 4 of these parameters, namely, the overall amplitude, initial phase and the orientation angles of the binary orbit. The maximization over the time of arrival (or, alternatively, over the time of final coalescence) of signal was carried out via FFTs. Estimates of computational costs involved in searching over the source-direction angles and the chirp mass were obtained for the simplistic case of Newtonian waveforms. In this work, we extend the coherent network analysis to the more realistic case of PN waveforms. A restricted PN waveform depends on individual masses of the companions instead of the combined chirp mass. This increases the number of parameters by one. We estimate, in general, the costs involved in searching over the masses as well as the source-direction angles for realistic network configurations. Finally, we describe a coincidence network detection strategy and compare costs incurred in it with those in the coherent detection strategy.

\section{Restricted post-Newtonian signal at the network}

The signal $s^{I}(t)$ at the constituent $I$-th detector of the network is given by [2]

$$
s^{I}(t)=2 \kappa \Re\left[\left(E_{I}^{*} S^{I}\right) e^{i \delta_{c}}\right],
$$

where $\kappa$ is the overall amplitude that depends on the fiducial frequency $f_{s}$ and the masses of the binaries. $\delta_{c}$ is the phase of the waveform at the time of final coalescence. The extended beam-pattern functions of the $I$-th detector, $E_{I}$, depend on the sourcedirection angles, $\{\theta, \phi\}$, the orbit orientation angles, $\{\epsilon, \psi\}$, the $I$-th detector orientation,

$\left(\alpha_{(I)}, \beta_{(I)}, \gamma_{(I)}\right)$, and the sensitivity, $g_{(I)}$, of the detector to the incoming signal. Finally, $S^{I}(t)$ is a normalized complex signal such that in the stationary-phase approximation (SPA) its Fourier transform (FT) is

$$
\tilde{S}^{I}\left(f ; t_{c}, \xi\right)=\frac{2}{g_{(I)}} \sqrt{\frac{2}{3 f_{s}}}\left(\frac{f}{f_{s}}\right)^{-7 / 6} \exp \left[i \Psi_{(I)}\left(f ; f_{s}, t_{c}, \xi\right)\right]
$$


for positive frequencies. Above, the phase of the 2.5 restricted PN waveform at the $I$-th detector is the scalar

$$
\Psi_{(I)}\left(f ; f_{s}, t_{c}, M, \eta, n_{3}, n_{1}\right)=\vartheta^{\mu} \xi_{(I) \mu}\left(f ; f_{s}\right),
$$

with the parameters $\vartheta^{\mu}$ consisting of the final-coalescence time $t_{c}$, the total mass $M$, the mass ratio $\eta\left(:=m_{1} m_{2} / M^{2}\right)$ and the source-direction described by two components $n_{1}$ and $n_{3}$ of unit vector $\hat{n}$ pointing to the source. Given below are $\vartheta^{\mu}$ and $\xi_{(I) \mu}$ :

$$
\begin{aligned}
\vartheta^{0} & =2 \pi f_{s} t_{c} ; & \xi_{(I) 0} & =\left(\frac{f}{f_{s}}\right) \\
\vartheta^{1} & =\frac{3}{128 \eta}\left(\pi M f_{s}\right)^{-5 / 3} ; & \xi_{(I) 1} & =\left(\frac{f}{f_{s}}\right)^{-5 / 3} \\
\vartheta^{2} & =\frac{1}{128 \eta}\left(\frac{3715}{252}+\frac{55}{3} \eta\right)\left(\pi M f_{s}\right)^{-1} ; & \xi_{(I) 2} & =\left(\frac{f}{f_{s}}\right)^{-1} \\
\vartheta^{3} & =-\frac{3 \pi}{8 \eta}\left(\pi M f_{s}\right)^{-2 / 3} ; & \xi_{(I) 3} & =\left(\frac{f}{f_{s}}\right)^{-2 / 3} \\
\vartheta^{4} & =\frac{3}{128 \eta}\left(\frac{15293365}{508032}+\frac{27145}{504} \eta+\frac{3085}{72} \eta^{2}\right)\left(\pi M f_{s}\right)^{-1 / 3} ; & \xi_{(I) 4} & =\left(\frac{f}{f_{s}}\right)^{-1 / 3} \\
\vartheta^{5} & =\frac{1}{128 \eta}\left(\frac{38645}{252}+5 \eta\right) \pi ; & \xi_{(I) 5} & =\ln \left[\frac{f}{f_{s}}\right] \\
\vartheta^{6} & =2 \pi n_{3} ; & \xi_{(I) 6} & =z_{(I)}\left(\frac{f}{f_{s}}\right) \\
\vartheta^{7} & =2 \pi n_{1} ; & \xi_{(I) 7} & =x_{(I)}\left(\frac{f}{f_{s}}\right)
\end{aligned}
$$

Here, $x_{(I)}$ and $z_{(I)}$ are, respectively, the $x$ and $z$ coordinate values (in units of $c / f_{s}$ ) of the location of the $I$-th detector in a fiducial reference frame.

\section{Number of templates}

In this section, we estimate the number of templates required to search over the parameter space. In Ref. [4], we showed that for a given pair of source-direction angles $(\theta, \phi)$, the network likelihood ratio, when maximized over the overall amplitude, $\delta_{c}, \epsilon$, and $\psi$, gives the network detection statistic. Numerical maximization of the statistic over the rest of the parameters, namely, masses and source-direction angles is performed by using a template bank. We estimate the number of templates by calculating the volume of the parameter space of interest obtained by the computing metric on the manifold and dividing by the size of each template. When the network statistic is dependent on the parameters solely through the difference between the parameter values of the signal and the template, then the metric on the parameter manifold is flat and, hence, the template placement is uniform.

It is well known that with $\mathrm{PN}$ order $>1$, the metric on the manifold is not flat. The Tanaka-Tagoshi [5] coordinates provide a convenient and an elegant way to carry out further analysis. The salient feature of these coordinates is to make the metric Euclidean on a flat manifold, which is an approximation to the actual manifold. Also 
the coordinate volume of the parameter space in these coordinates is same as the proper volume which immediately gives the number of templates as has been described above.

\section{Computational costs}

In this section, we estimate the cost involved in numerically searching over the rest of the parameter space mentioned above. This cost has two important components:

(i) The cost involved in FTs: MLD technique requires to cross-correlate the data with all possible templates in the rest of the parameter space involving mass parameters and the direction angles. Since information of the direction angles is encoded in time delays, network correlation vectors for templates differing in direction angles can be constructed by combining the correlation outputs from different detectors with appropriate time-delays as described in [2]. Thus, the cost involved in FTs is equal to the number of computational operations required in searching over the intrinsic parameters, in our case, the two masses of the binary.

(ii) The cost involved in scanning the time-delay window: The optimal statistic needs to be evaluated by combining the correlation vectors with appropriate time-delays.

Consider a network of $N_{D}$ detectors. Let $N$ be the number of sampled points in a data train at each constituent detector. If the templates are stored in memory, then the computing cost in FTs is $6 N_{D} N n_{X_{1}-X_{2}} \log _{2} N$, where $n_{X_{1}-X_{2}}$ reflects the number of templates in mass parameters. The number of floating point operations to construct a network statistic for one pair of direction angles is $8 N_{D}$. If $n_{t o t}$ is the total number of templates then the total computational cost is

$$
C=2 N_{D} N\left(8 n_{t o t}+3 n_{X_{1}-X_{2}} \log _{2} N\right) .
$$

Online data processing requires the data processing rate should be equal to the data acquisition rate. Thus the length of the data which is effectively processed is equal to the length of the zero padding. We obtain the online computational speed by dividing the cost by the length of the padding interval. We use the analytical fits to the noise curves of LIGO and VIRGO which we enlist in Table 11. We tabulate the results for

Table 1. Analytical fits (for positive frequencies) to noise power spectral densities, $2 s_{h}(f)$, of the interferometric detectors studied in this paper [7]. We take $s_{h}(f)$ to be infinite below the seismic cut-off frequency $f_{s}$. We choose the high frequency cut-off, $f_{c(I)}$, to be $800 \mathrm{~Hz}$ for all $I$.

\begin{tabular}{lcrr}
\hline Detector & Fit to noise PSD, $10^{46} \times s_{h}(f) / \mathrm{Hz}^{-1}$ & $f_{0}(\mathrm{~Hz})$ & $f_{s}(\mathrm{~Hz})$ \\
\hline VIRGO & $3.24\left[\left(6.23 f / f_{0}\right)^{-5}+2\left(f_{0} / f\right)+1+\left(f / f_{0}\right)^{2}\right]$ & 500 & 20 \\
LIGO I & $9.0\left[\left(4.49 f / f_{0}\right)^{-56}+0.16\left(f / f_{0}\right)^{-4.52}+0.52+0.32\left(f / f_{0}\right)^{2}\right]$ & 150 & 30 \\
\hline
\end{tabular}

various idealistic as well as realistic networks in Table 2. For the case of real network of LIGO-VIRGO [6] with their respective noises, we estimate the average number of templates for most of the astrophysical range of $\epsilon$ and $\psi$ to be $n_{t o t} \sim$ few times $\times 10^{10}$ 
Table 2. Number of templates, computational costs, and online computing speeds required for a search using specific networks. The detector networks are labeled as $I$ for a single detector, III for three identical detectors with identical orientations placed on Earth's equator forming an equilateral triangle. The detector $X_{D}$ denotes a detector with LIGO-I noise at the location of the detector $D$. The letters $L, H, V$, $T$, and $A$ denote, LIGO detector at Louisiana, LIGO detector at Hanford (of $4 \mathrm{~km}$ arm-length), VIRGO, TAMA and AIGO sites, respectively. We assume LIGO-I noise for both the LIGO detectors. We present results for lower mass limits of $0.5 M_{\odot}$ and $1.0 M_{\odot}$. The maximum length of the $2.5 \mathrm{PN}$ chirp is 96.5 secs and 306 secs for minimal mass limits of $1 M_{\odot}$ and $0.5 M_{\odot}$ respectively. We assume fiducial frequency $f_{s}=30$ $\mathrm{Hz}$ except for the $L V$ case. We consider data trains of $1100 \mathrm{sec}$. for $0.5 M_{\odot}$ and 400 sec. for $1.0 M_{\odot}$ sampled at $2 \mathrm{kHz}$ so that $N \sim 10^{6}$. For the $L V$ network, the length of the longest chirp is $\sim 284 \mathrm{sec}$. for $1.0 M_{\odot}$ and $\sim 900 \mathrm{sec}$. for $0.5 M_{\odot}$. The number of points in the data train $\sim 10^{6}-10^{7}$. The mismatch is taken to be $3 \%$.

\begin{tabular}{cccccc}
\hline $\begin{array}{c}\text { Network } \\
\text { configuration }\end{array}$ & $\begin{array}{c}\text { mass limit } \\
\left(M_{\odot}\right)\end{array}$ & $\begin{array}{c}n_{\text {tot }} \\
\left(\times 10^{7}\right)\end{array}$ & $\begin{array}{c}n_{X_{1}-X_{2}} \\
\left(\times 10^{4}\right)\end{array}$ & $\begin{array}{c}C_{\text {tot }}\left(\times 10^{14}\right) \\
(\mathrm{fl}-\mathrm{pt} \text { ops })\end{array}$ & $\begin{array}{c}S \\
(\mathrm{Gflops})\end{array}$ \\
\hline$I$ & 0.5 & 0.0214 & 10.7 & 0.3 & 0.37 \\
& 1.0 & 0.0042 & 2.1 & 0.02 & 0.06 \\
$L H$ & 0.5 & 0.81 & 16 & 37.5 & 4.7 \\
& 1.0 & 0.16 & 3.2 & 0.37 & 1.2 \\
$L X_{V}$ & 0.5 & 2.2 & 16 & 8.7 & 19 \\
& 1.0 & 0.44 & 3.2 & 0.72 & 2.35 \\
$L X_{T}$ & 0.5 & 2.67 & 16 & 10 & 12 \\
& 1.0 & 0.52 & 3.2 & 0.82 & 2.7 \\
$L X_{A}$ & 0.5 & 1.7 & 16 & 6.8 & 15 \\
& 1.0 & 0.68 & 3.2 & 1 & 3.36 \\
$L V$ & 0.5 & 14.1 & 93 & 160 & 67 \\
& 1.0 & 2.7 & 19 & 11 & 12 \\
$I I I$ & 0.5 & $1.5 \times 10^{3}$ & 16 & $7.7 \times 10^{3}$ & 9700 \\
& 1.0 & $2.9 \times 10^{2}$ & 3.2 & $5.5 \times 10^{2}$ & 180 \\
\hline
\end{tabular}

for lower mass limit of $0.5 M_{\odot}$. We take data trains of length $3000 \mathrm{sec}$. corresponding to the longest chirp of $\sim 900 \mathrm{sec}$. for VIRGO. Taking a sampling rate of $2 \mathrm{kHz}$, the data must be processed in $2100 \mathrm{sec}$. The online data processing demands a computational speed of few thousands Tflops.

Here, we note that for networks with $N_{D} \geq 3$, the computational cost required to construct optimal network statistic while searching over the source-direction angles overshoots the FT costs. As a result, the computational requirements are beyond the reach of the current technology for a flat search.

\section{Coincident Search}

To focus on the essential aspects of a coincidence search strategy, we consider the simplistic case of a network comprising of detectors with identical noises and 
orientations, but with arbitrary locations. The network detection statistic in such a search is taken to be the minimum element in $\left\{\left|\mathcal{C}^{1}\right|, \ldots,\left|\mathcal{C}^{N_{D}}\right|\right\}$, where $\mathcal{C}^{I}$ is the single detector statistic evaluated on the data of the $I$-th detector (see Ref. [8). Therefore, unlike a coherent search, a coincident search involves first establishing threshold-crossing by the single-detector detection statistic in each of the detectors in a network. Furthermore, claiming a detection by the network requires that the parameters corresponding to the threshold-crossing templates lie within error intervals of one another, such that they can be consistently ascribed to a a single astrophysical event. This requirement alone immediately implies that, even in this simplistic network the computational cost in a coincident search is larger than $N_{D} C_{1}$, where $C_{1}$ is the computational cost for a single detector search.

To ascertain exactly how large this cost is, we first describe for a network of two identical detectors our search algorithm, which is based upon a most powerful search and is not necessarily the cheapest computationally [9]:

(i) Filter the data $x^{1}(t)$ and $x^{2}(t)$ from the two detectors, respectively, with a bank of single-detector templates to draw two separate lists of threshold crossers. Label these "candidate events" $E_{i}^{1}=E\left(t_{i}^{a 1} ; \boldsymbol{\vartheta}_{i}^{1}\right)$ and $E_{j}^{2}=E\left(t_{j}^{a 2} ; \boldsymbol{\vartheta}_{j}^{2}\right)$, respectively, where $t_{i}^{a 1}\left(t_{i}^{a 2}\right)$ denotes the time of arrival of event $i$ at detector 1 (2), and $i=1,2, \ldots, m, j=1,2, \ldots, n$. Note that $m \neq n$, in general. Also, $\boldsymbol{\vartheta}_{i}^{1}$ denotes the template-parameter vector characterizing event $i$ at detector 1 . The above nomenclature is suited to handle the possibility of two or more templates triggering off simultaneously, say, on the data from detector 1 . In such a case, one will have more than one event with $t_{i-1}^{a 1}=t_{i}^{a 1}=t_{i+1}^{a 1}$, but with $\boldsymbol{\vartheta}_{i-1}^{1} \neq \boldsymbol{\vartheta}_{i}^{1} \neq \boldsymbol{\vartheta}_{i+1}^{1}$.

(ii) "Time window" veto: Let detector 1 have a smaller number of candidate events than detector 2. With each $E_{i}^{1}$ associate a set $W^{2}\left(t_{i}^{a 1} ; \tau_{c}^{12}{ }_{i j}\right)$ of candidate events $E_{j}^{2}$, such that $t_{i}^{a}-\tau_{c i j}^{12} \leq t_{j}^{a} \leq t_{i}^{a}+\tau_{c i j}^{12}$. Here, $\tau_{c i j}^{12}$ is the sum of the light-travel time between the two detectors and the sum of magnitudes of the estimated errors in their arrival times at detectors 1 and 2. Note that an event $E_{j}^{2}$ may appear in more than one set. That is, it may happen that $E_{j}^{2} \in W^{2}\left(t_{i}^{a 1} ; \tau_{c i j}^{12}\right) \cap W^{2}\left(t_{k}^{a 1} ; \tau_{c k j}^{12}\right)$, where $i \neq k$. Discard from the lists those $E_{j}^{2}$ that do not belong to any $W^{2}\left(t_{i}^{a 1} ; \tau_{c i j}^{12}\right)$.

(iii) "Parameter window" veto: Compute the covariance matrix in the parameter space around $E_{i}^{1}$ and around each event in $W^{2}\left(t_{i}^{a 1} ; \tau_{c i j}^{12}\right)$ from the ambiguity function [3]. Estimate the parameter error, $\Delta \boldsymbol{\vartheta}_{i}^{1}\left(\Delta \boldsymbol{\vartheta}_{j}^{2}\right)$, to be the square-root of the variance of the parameter $\boldsymbol{\vartheta}_{i}^{1}\left(\boldsymbol{\vartheta}_{j}^{2}\right)$ derived from this matrix. Discard events in $W^{2}\left(t_{i}^{a 1} ; \tau_{c i j}^{12}\right)$ that have $\left|\vartheta_{j}^{2 \mu}-\vartheta_{i}^{1}{ }^{\mu}\right|>\left|\Delta \vartheta_{j}^{2 \mu}\right|+\left|\Delta \vartheta_{i}^{1}{ }^{\mu}\right|$ for each parameter index $\mu$.

The pairs of candidate events surviving the above vetoes are the "detected" events. A more sophisticated approach involving further vetoes of the type discussed in Ref. [10] will be studied elsewhere.

The above steps explicitize the computational costs, over and above that of $N_{D} C_{1}$, that are necessary in a coincidence detection, but are often glossed over: Extra costs are involved in computing parameter errors and implementing vetoes based on them. These 
costs obviously scale as the number of the candidate events in each detector (whereas, the cost in a coherent search is independent of it). These counts, in turn, depend on the value of the detection threshold and, therefore, on the false-alarm probability. The number of floating point operations (Flop) needed to estimate the error in a parameter, $\vartheta_{i}^{I}{ }^{\mu}$, is close to that involved in taking the second derivative of $\mathcal{C}^{I}$ with respect to $\vartheta_{i}^{I \mu}$. Using the discrete version of second derivative the number of Flop involved $\sim 10 C_{1}$. Therefore, in an 8 dimensional parameter space (based on the independent parameters $\left(r, \delta_{c}, \vartheta^{0}, \ldots, \vartheta^{5}\right)$, the number of Flop required to estimate parameter errors for all candidate events is about $80 C_{1} \times \sum_{I=1}^{N_{D}} N_{I}$, where $N_{I}$ is the number of candidate events in detector $I$. Additional operations required to compare the parameter values across detectors (using the inequality given in step (iii) above) and veto events scales as $\prod_{I=1}^{N_{D}} N_{I}$, which is a small fraction of the total cost for $N_{D}=\mathcal{O}(1)$ and $N_{I}=\mathcal{O}(10)$. Thus, neglecting this last contribution, the total number of Flop scales as:

$$
N_{D} C_{1}+80 C_{1} \sum_{I=1}^{N_{D}} N_{I} .
$$

For comparison with the coherent search costs, we take $N_{I}=10^{2}$ in $N \simeq 10^{6}$ data points in each of the 3 detectors in a network. For, a minimum mass of $0.5 M_{\odot}$, Table 2 shows that $C_{1}=0.3 \times 10^{14}$. Thus, for network configuration $I I I$, the total number of Flop in a coincident search is about $7.2 \times 10^{17}$ which is very close to $C_{\text {tot }}=7.7 \times 10^{17}$ for a coherent search. One may argue that it is possible to reduce $N_{I}$ in each detector by using additional vetoes of the type adopted in Ref. [10]. Such steps will surely reduce the contribution from the second term in eq.(6). Nevertheless, the additional costs in implementing such vetoes is very large as well and must be explored in more detail.

It is easy to see that with more events or more detectors, the cost related to Eq. (6) can only rise. With future detectors, where the detection thresholds will be lower than present ones owing to their higher sensitivity, the number of events with larger signalto-noise ratios will increase, consequently, increasing the computational cost further of a coincidence search.

\section{Conclusion}

As shown in Table 2., the computational cost in a coherent search rises markedly in going from $N_{D}=1$ to 3 . This is expected because the number of parameters and, therefore, the parameter volume accessible to a search increases from 5 (for $N_{D}=1$ ) to 9 (for $N_{D}=3$ ). Indeed, for $N_{D} \geq 3$ the cost required to search over source-direction angles overshoots that required for the FFTs. Beyond $N_{D}=3$, the computational cost in a coherent search, however, stabilizes. This must be contrasted with the cost behavior in a coincidence search, where it continues to increase with $N_{D}$. Specifically, given a network of identical detectors and a false-alarm probability, for a low enough detection threshold, a coincidence search will cost more than a coherent search for $N_{D}>1$. In

either search, the computational costs are very large and, hence, call for investing in the exploration of more efficient search techniques, such as hierarchical strategies. 


\section{Acknowledgments}

AP would like to thank CSIR, India for SRF.

[1] T. Damour, B. R. Iyer and B. S. Sathyaprakash, Phys. Rev. D 63, 044023 (2001).

[2] A. Pai, S. V. Dhurandhar and S. Bose, Phys. Rev. D 64, 042004 (2001).

[3] C. W. Helstrom, Statistical Theory of Signal Detection (Pergamon Press, London, 1968).

[4] S. Bose, A. Pai, and S. V. Dhurandhar, Int. J. Mod. Phys. D 9, 325 (2000). (gr-qc/0002010)

[5] T. Tanaka and H. Tagoshi, Phys. Rev. D 62, 0822001 (2000).

[6] B. Allen, Gravitational wave detector sites, gr-qc/9607075.

[7] Analytical fits obtained by private communication with Dr. B. S. Sathyaprakash.

[8] S. Finn, Phys. Rev. D63, 102001 (2001). (gr-qc/0010033)

[9] This algorithm extends the one described in Ref. [8].

[10] B. Allen et al, Phys. Rev. Lett. 831498 (1999). (gr-qc/9903108) 